\title{
EFEITO DA SOLDAGEM NO COMPORTAMENTO MECÂNICO DO AÇO INOXIDÁVEL AISI 304 APLICANDO A CORRELAÇÃO DIGITAL DE IMAGENS*
}

Ycaro Jorge Maia da Costa ${ }^{1}$ José Máspoli Ferreira Pereira ${ }^{1}$ Rodrigo Nogueira de Codes ${ }^{1}$

\section{Resumo}

O desenvolvimento de estruturas com dimensionamento confiável requer o monitoramento de seus materiais, entretanto alguns processos de fabricação muitas vezes induzem modificações em seu comportamento mecânico. Isso faz com que as estruturas sejam menos confiáveis podendo causar redução da vida útil. Um dos processos onde isso acontece é a soldagem. Seu aporte térmico, modifica a microestrutura do material de tal forma a gerar três regiões mecanicamente distintas na junta soldada: Metal de Solda (MS), Zona Afetada pelo Calor (ZAC) e Metal de Base (MB), fazendo com que haja concentração de tensão na região afetada. Este trabalho se deteve em analisar o comportamento mecânico de uma junta soldada de aço inoxidável AISI 304 em comparação com o mesmo material não-soldado através da identificação dos gradientes de deformação, utilizando da Correlação Digital de Imagens (CDI) aplicada ao ensaio de tração. Os resultados provaram a capacidade da técnica de CDI em identificar campos de deformação mesmo em materiais anisotrópicos, como uma junta soldada.

Palavras-chave: Correlação Digital de Imagens; Soldagem MIG; Comportamento mecânico.

\section{THE EFFECT OF WELDING IN THE MECHANICAL BEHAVIOR OF STAINLESS STEEL AISI 304 APPLYING DIGITAL IMAGE CORRELATION}

\section{Abstract}

The development of structures with optimized design requires the monitoring of their materials, though some manufacturing processes often induce changes in their mechanical behavior, this makes the structures are less reliable and may cause reduced service life. One of the cases where this happens is welding. Its heat input, changes the microstructure of the material in such a way to generate three mechanically distinct regions in the welded joint: Weld Metal (WM), Heat Affected Zone (HAZ) and Base Metal (MB), so that there is concentration tension in the affected region. This work is aimed to examine the mechanical behavior of a welded joint of AISI 304 stainless steel compared with the same non-welded material by identifying the strain gradients using the Digital Image Correlation technique (DIC) applied to the tensile test. The results proved the ability of the CDI technique to identify strain fields even in anisotropic materials, such as a welded joint.

Keywords: Digital Image Correlation; GMAW welding; Mechanical behavior.

1 Departamento de Ciências Ambientais e Tecnológicas - DCAT, Universidade Federal Rural do Semi-Árido - UFERSA, 59.625-900, Mossoró, RN, Brasil. 


\section{INTRODUÇÃO}

O avanço na engenharia promoveu a necessidade do projeto de estruturas dimensionadas de forma otimizada. Esse contexto fez com que houvesse a busca por alternativas de monitoramento do comportamento mecânico de materiais.

Tradicionalmente o ensaio de tração se destaca por fornecer a maior quantidade de informações sobre o comportamento mecânico dos metais em comparação com outros ensaios mecânicos. Entretanto, este avalia o comportamento mecânico do material de forma global.

O projeto de estruturas metálicas pode envolver vários processos de fabricação, dentre eles: soldagem, usinagem, tratamentos térmicos, químicos, termoquímicos e endurecimento por deformação decorrentes da construção e montagem. Estes processos podem induzir a modificação deste comportamento mecânico global num material.

Assim a previsão do desempenho da estrutura através do ensaio mencionado e/ou de outros ensaios mecânicos se torna mais difícil, pois certas regiões da estrutura podem concentrar tensões, ter propriedades mecânicas do material modificadas de forma pontual em decorrência de transformações microestruturais, e em virtude disso, apresentarem campos de deformação não homogêneos. A análise do comportamento mecânico em muitos casos desse tipo não é possível, devido a restrições geométricas ou se torna caro utilizar vários extensômetros (strain gauges). [1],[2].

Estas dificuldades existem quando se estuda o comportamento mecânico de juntas soldadas. Segundo Galvis e Hormoza [3] e Kumar e Shahi [4] devido ao fato de a dissipação de calor neste processo acontecer principalmente por condução a partir da região onde ocorreu a fusão do metal consumível para o restante do material da junta soldada, são gerados gradientes térmicos na união. Durante o resfriamento esses gradientes provocam um tratamento termomecânico violento que inclusive causa deformações induzidas no material, segundo Chen et al. [5] e consequentemente acarretam gradientes microestruturais e mecânicos.

Existem três regiões microestrutural e mecanicamente distintas numa junta soldada: metal de base (MB), que é a região que o calor não é suficiente para gerar modificações em relação às condições originais; metal de solda (MS), que é a mistura solidificada de metal de adição e metal de base fundidos durante a soldagem e a zona afetada pelo calor (ZAC) que se divide em duas porções, ambas sofrem recristalização, uma pequena região de grãos refinados e outra maior com uma microestrutura de grãos grosseiros e alongados, o que faz com que haja um incremento em ductilidade e redução em resistência [6],[7].

Como relatado por Saranath et al.[1], a presença de três regiões mecanicamente distintas numa junta soldada faz com que o comportamento mecânico de deformação seja anisotrópico. Deste modo, faz-se necessária a aplicação de uma técnica que seja capaz de detectar e mostrar este gradiente de deformação durante o ensaio de tração, evitando assim a ocorrência de falhas em estruturas soldadas.

O conhecimento do comportamento de deformação de juntas soldadas pode evitar o superdimensionamento de estruturas, com a consequente redução de coeficientes de segurança, pois se fornece mais confiabilidade ao projeto. Adicionalmente, há a redução do volume de material utilizado e os custos de fabricação.

A técnica de Correlação Digital de Imagens (CDI) se destaca pela investigação de campos de deslocamento e de deformação. A técnica se baseia em imagens para 
identificar deslocamentos e através destes calcular deformações, por meio de uma comparação entre estados não-deformados e deformados.

O presente trabalho se deteve em mostrar a capacidade da técnica de Correlação Digital de Imagens em determinar o comportamento de campos de deformações mecanicamente isotrópicos e anisotrópicos de corpos de prova soldado e nãosoldado, através do ensaio de tração.

A técnica de CDI, foi desenvolvida, inicialmente, por pesquisadores da Universidade da Carolina do Sul, nos Estados Unidos, durante os anos 80. Ela foi inicialmente proposta à comunidade científica como método para análise gráfica da deformação de materiais, nos trabalhos de: Chu et al. [8], Peters e Ranson, [9], Sutton et. al. [10],[11] e Bruck et al. [12], atualmente ela é utilizada amplamente em pesquisas que estudam o comportamento mecânico de deformações dos mais variados materiais, sob diversas condições, com análise em duas e três dimensões.

O procedimento experimental da técnica CDI consiste de três etapas básicas: limpeza da amostra para a aplicação de uma textura em escala de cinza adquirida através de uma pintura da superfície da amostra, registro das imagens com uma frequência de aquisição pré-determinada durante a deformação da amostra e processamento das imagens em um programa CDI para adquirir os dados dos campos de deslocamento e deformação [13][14][15].

A textura em escala de cinza na superfície da amostra é conseguida através da aplicação de uma nuvem de tinta spray branca sobre uma superfície pintada homogeneamente de preto [16]. A nuvem de tinta branca deve fazer com que haja uma distribuição aleatória das gotículas brancas sobre a superfície preta formando a textura em escala de cinza como mostrado na figura 1-A.

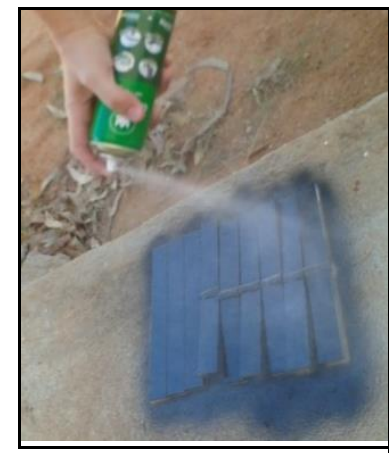

Figura 1-A -

Aplicação da textura em escala de cinza

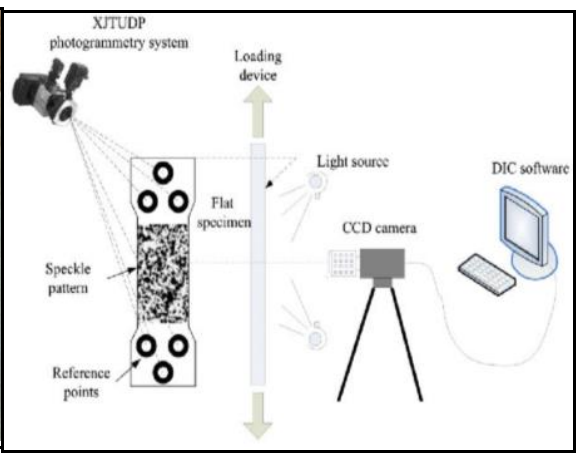

Figura 1-B - $\quad$ Aparato experimental para ensaio de tração aplicando a técnica CDI [17]
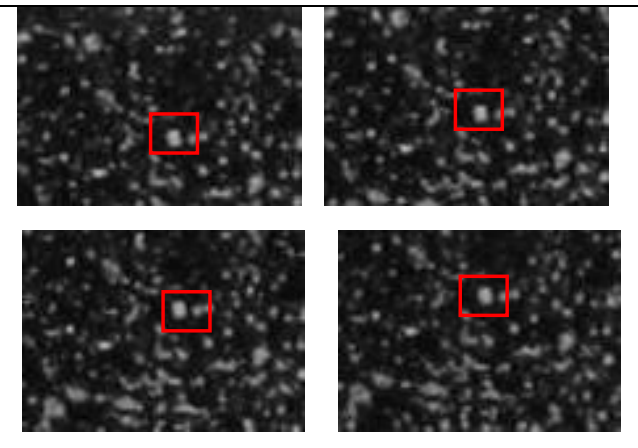

Figura 1-C - Deslocamento de um ponto de controle e sua área de interesse (5s entre cada imagem).

Um esquema típico de da aplicação da técnica Correlação Digital de Imagens é mostrado na figura 1-B [17].

Durante o Processamento Digital de Imagem (PDI), o programa CDI converte as informações visuais contidas na textura em informações descritivas na forma de um modelo matemático. A textura em escala de cinza de cada imagem funciona como uma função $\boldsymbol{f}(\boldsymbol{x}, \boldsymbol{y})$, onde o domínio da função é dado pelas coordenadas $(\boldsymbol{x}, \boldsymbol{y})$ da imagem digital em pixels e a imagem $\boldsymbol{f}$ representa a luminosidade de cada posição. O conjunto de valores em escala monocromática constitui uma matriz de luminosidade da imagem [13], [18].

Os deslocamentos são calculados através da comparação entre as matrizes luminosidade de uma imagem de referência e imagens. Cada valor da matriz luminosidade da imagem de referência representa um elemento de área (em 
vermelho na figura 1-C que é reconhecido pelo programa $C D I$ através de um ponto de controle, fazendo com que o elemento de área seja identificado mesmo após ocorrer o deslocamento em uma imagem deformada [19], [20].

A figura 1-C mostra a sequência de deslocamento de um ponto de controle, e a movimentação de sua área de interesse conforme ocorre a deformação do material.

Para o programa a função que corresponde ao elemento da matriz de luminosidade da imagem de referência, $\boldsymbol{I} \boldsymbol{o}(\boldsymbol{x}, \boldsymbol{y})$, que será correlacionada com a da imagem deformada, $\boldsymbol{I n}=\left(\boldsymbol{x}^{\prime}, \boldsymbol{y}^{\prime}\right)$, satisfaz a relação: $\boldsymbol{I} \boldsymbol{o}(\boldsymbol{x}, \boldsymbol{y})=\boldsymbol{I n}(\boldsymbol{x}+\boldsymbol{u n}, \boldsymbol{y}+\boldsymbol{v n})$. Onde $\boldsymbol{u n}$ e vn representam os deslocamentos na abcissa e ordenada respectivamente [18].

Os deslocamentos un e vn são determinados pelo coeficiente de correlação cruzada $\boldsymbol{C}$ :

$$
C=\frac{\sum_{u n, v n}(x, y) \cdot I_{n}(x+u n, y+v n)}{\sqrt{\sum_{u n, v n} I_{o}(x, y)^{2} \cdot \sum_{u n, v n n} I_{n}(x+u n, y+v n)^{2}}}
$$

O coeficiente de correlação cruzada é adotado quando seu valor em função dos deslocamentos é máximo, assim $\boldsymbol{C}$ é função dos valores de deslocamentos un e vn e de seus gradientes.

Hild e Roux [14],[15] desenvolveram um programa (Correli Q4 ${ }^{\circledR}$ ) com algoritmos de Correlação Digital de Imagens implementados na plataforma MATLAB ${ }^{\circledR}$. Seus trabalhos constataram seu potencial para mensurar campos de deslocamento e deformação, bem como determinar propriedades mecânicas dos materiais. Além disso, o programa mostrou a capacidade de determinar deslocamentos em escala de sub-pixels, isto comprova a confiabilidade dos resultados de ensaios obtidos através do uso do Correli Q4.

Além de apresentar os campos de deslocamento e deformação em interface gráfica, - Correli Q4 tem como grande vantagem em relação a outros programas CDI, o recurso de análise de textura antes de iniciar o ensaio.

A técnica CDI foi utilizada para mensurar tensões residuais e evolução de deformação durante o procedimento de soldagem TIG/GTAW como mostrado no estudo de Strycker et al. [21]. Os autores conseguiram provar a eficácia deste experimento, comparando os resultados da evolução do campo de deformação através da técnica CDI com os de extesômetros elétricos.

$\mathrm{Hu}$ et al. [13] utilizaram a técnica de Correlação Digital de Imagens em três dimensões para identificar o comportamento de deformação induzida na soldagem de chapas de aço inoxidável, durante a soldagem, ao fim do processo e durante 0 resfriamento. Os autores relataram que a técnica conseguiu através de sua interface representar de forma ilustrativa os campos de deformação decorrentes dos gradientes térmicos do processo de soldagem.

Jin et al. [22] utilizaram a técnica de CDI para análise de deformações devido às tensões térmicas referentes a um conjunto metálico composto por uma placa circular de alumínio e um anel de titânio que com o aquecimento entravam em interferência gerando o campo de deformações.

Rêgo e Codes [23] aplicaram a técnica CDI usando o Correli Q4 durante ensaios de tração, com o intuito de investigar o comportamento viscoelástico de materiais termoplásticos submetidos a concentradores de tensão, através da variação da taxa de deformação dos ensaios. Dessa maneira foi possível observar por meio de sua interface gráfica o comportamento do campo de deformações na região de concentração de tensão. 
Codes et al. [24] utilizaram o Correli Q4 para identificar a propagação de bandas oriundas do efeito Potervin-Le Chatelier, em amostras de AA 5182, durante o ensaio de tração.

\section{MATERIAIS E MÉTODOS}

Foram fabricados seis corpos de prova nas condições soldado (ensaios $A 1, A 2$ e A3) e não-soldado (B1, B2 e B3), de acordo com a norma ISO 6892, para ensaio de tração à temperatura ambiente. As dimensões foram de $25,4 \mathrm{~mm}$ da largura, $5 \mathrm{~mm}$ de espessura e $200 \mathrm{~mm}$ de comprimento. O material foi usinado a partir de barras chatas de aço inoxidável AISI 304, que segundo a certificação do fabricante possui composição química conforme a tabela 1.

Tabela 1. Composição química do aço inoxidável AISI 304

\begin{tabular}{lccccccccccc}
\hline Elemento & $\mathbf{C}$ & $\mathbf{S i}$ & $\mathbf{M n}$ & $\mathbf{P}$ & $\mathbf{C r}$ & $\mathbf{M o}$ & $\mathbf{N i}$ & $\mathbf{V}$ & $\mathbf{A l}$ & $\mathbf{C u}$ & $\mathbf{T i}$ \\
\hline$\%$ p. & 0,04 & 0,4 & 1,28 & 0,3 & 18,2 & 0,32 & 7,63 & 0,08 & 0,003 & 0,55 & 0,004 \\
\hline
\end{tabular}

Para confecção das juntas soldadas os corpos de prova foram cortados longitudinalmente no centro de seu comprimento através de um chanfro em "I" para posterior união em ambos os lados por soldagem MIG/GMAW. A soldagem foi realizada por um robô de deslocamento de tocha (Tartílope V1 figura 2-A). A máquina de solda utilizada foi de modelo ESAB LAI 407 com alimentador de arame acoplado (figura 2-B). A aquisição dos parâmetros de soldagem foi feita por um sistema Aq. Dados 7.02.

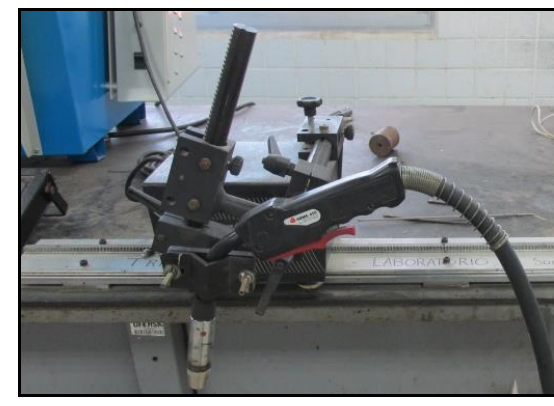

Figura 2-A - Tartílope V1

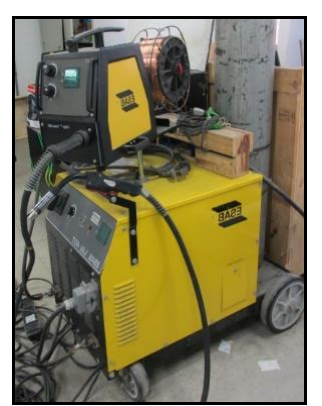

Figura 2-B - ESAB LAI 407

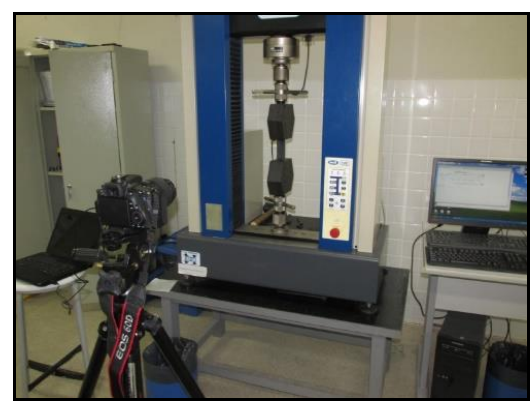

Figura 2-C - Aparato de Ensaio

O arame consumível utilizado possui especificação AWS ER308LSi. Usou-se o argônio puro como gás de proteção. Os parâmetros de soldagem utilizados foram: $195 \mathrm{~A}$ de corrente, $27 \mathrm{~V}$ de tensão, 20l/min de vazão de gás e um deslocamento da tocha de $30 \mathrm{~cm} / \mathrm{min}$. Estes parâmetros representaram um aporte térmico de $10,5 \mathrm{~kJ} / \mathrm{cm}$.

A soldagem procedeu-se com abertura e fechamento de arco em chapas adjacentes de mesma espessura e material do corpo de prova, como mostrado na figura 3-A, impedindo os fenômenos de variação de parâmetros durante esses processos, fazendo com que os cordões de solda tenham a melhor continuidade possível. 0 metal adjacente foi posteriormente retirado por serragem. Condição final do corpo de prova é ilustrada na figura 3-B.

Foi aplicada a textura em escala de cinza nos corpos de prova (figura 1-A) conforme[16]. A aquisição das imagens foi realizada remotamente por uma câmera 
Cannon EOS $60 \mathrm{D}$ através de um computador, com uma frequência de aquisição de uma imagem a cada 5 segundos.

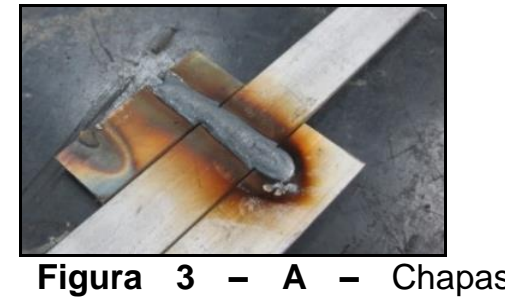

adjacentes ao corpo de prova.

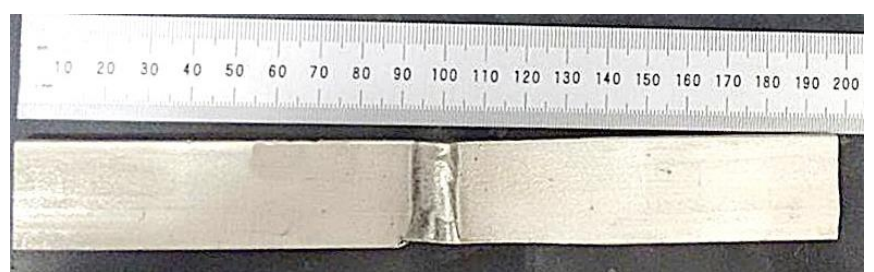

Figura 3 - B - Corpo de prova de aço inoxidável AISI 304 soldado.

Os ensaios foram realizados até a ruptura numa máquina universal de ensaios EMIC DL 10000 com capacidade máxima de $100 \mathrm{kN}$. Os comprimentos uteis de todos os corpos de prova foram de $115 \mathrm{~mm}$. Todos os ensaios foram realizados utilizando uma velocidade de deformação de $7,5.10^{-4} \mathrm{~s}^{-1} .0$ aparato experimental utilizado no ensaio é mostrado na figura 2-C Após os ensaios as imagens foram processadas e analisadas pelo software CDI Correli Q4.

\section{RESULTADOS E DISCUSSÃO}

Os resultados foram obtidos através do programa Correli Q4 são apresentados nesta seção. A figura 4 mostra a interface de resultados, onde é possível visualizar os campos de deslocamento e deformação nas duas direções do plano através de uma escala de cores, em que cada tom representa um valor do resultado obtido. Tais representações são: a deformação vertical, e a horizontal, o deslocamento vertical e o horizontal, a rotação e o erro. Esses dados são calculados através da comparação entre uma imagem de referência e uma imagem deformada a um determinado instante de tempo.

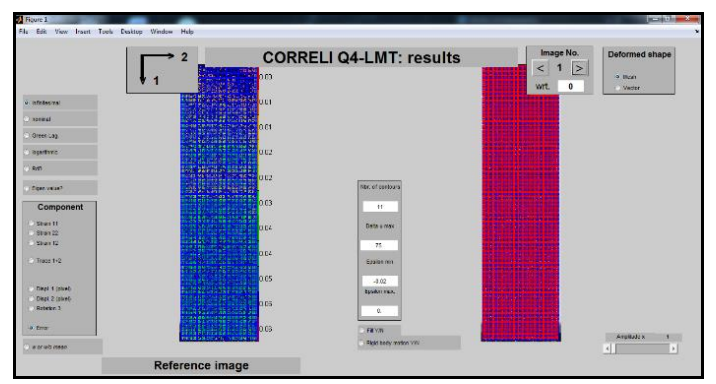

Figura 4 - Interface de visualização dos resultados Correli Q4

Este trabalho se ateve no estudo dos campos de deformação, pois estes permitem uma melhor observação do comportamento mecânico das regiões da junta soldada. A rotação também não é relevante neste caso pois os ensaios foram de tração uniaxial, portanto não será mostrada.

A escala de conversão milímetro-pixel foi calculada com base nas dimensões da imagem e do corpo de prova, tendo um resultado de $0,032 \mathrm{~mm} /$ pixel para ambos ensaios.

Os campos de deformação na direção 1 são mostrados na figura 5. A figuras 5-A e 5-B ilustram respectivamente os campos de deformação na direção 1 ensaios A2 e B1 nos instantes 580 e 1095s imediatamente antes da ruptura em relação à imagem de referência antes de qualquer deformação. 
Comparando as figuras $5-\mathrm{A}$ e $5-\mathrm{B}$, pode-se observar que a soldagem modificou consideravelmente o comportamento de deformação. Na primeira imagem é possível distinguir nitidamente MS, ZAC e MB, enquanto que na segunda observa-se uma deformação homogênea segundo a escala de cores.

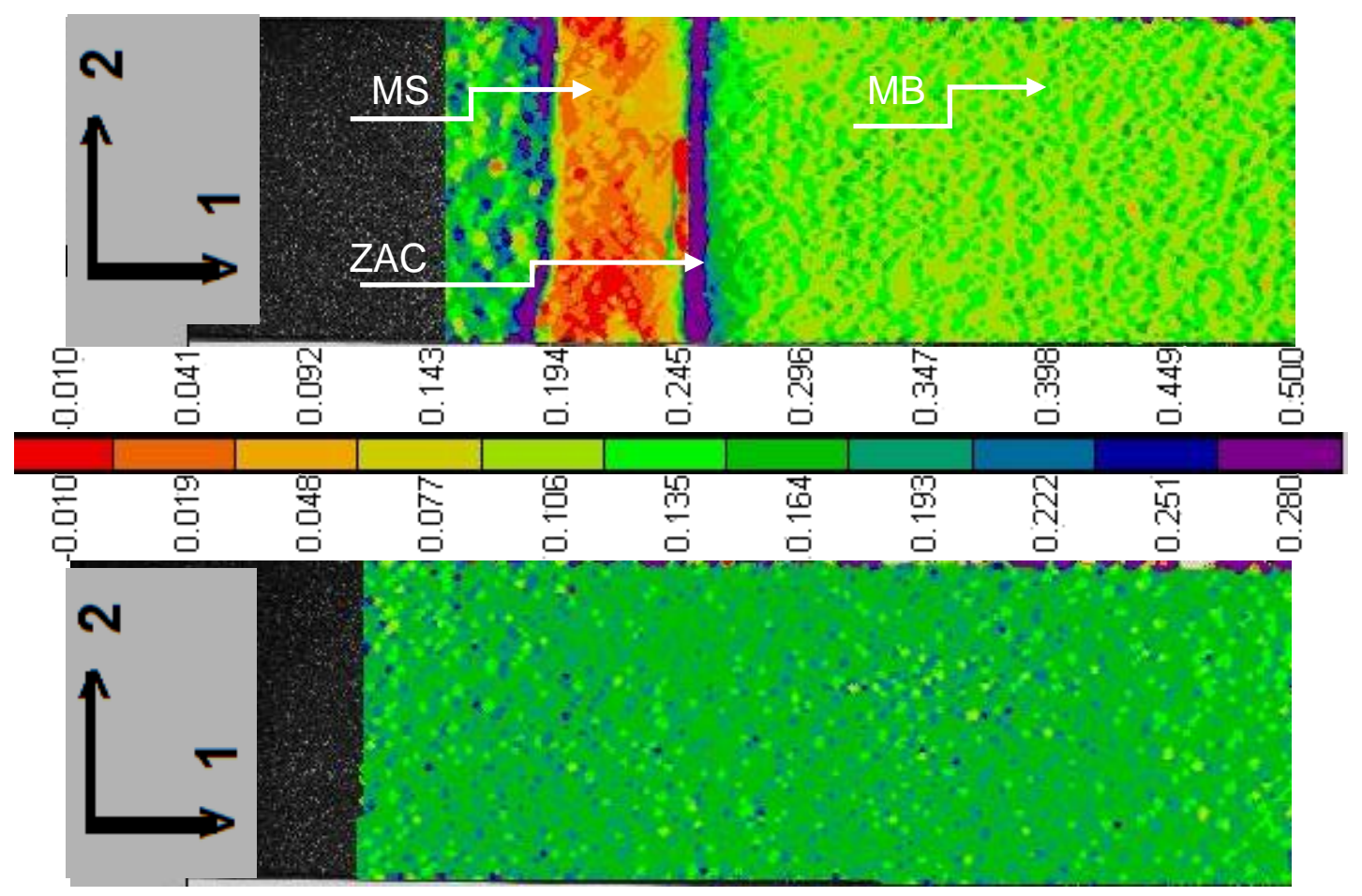

Figura 5-A - Campo de deformação na direção 1, amostra soldada A2 (580s).

Figura 5-B - Campo de deformação na direção 1, amostra não-soldada B1 (1095s).

Como esperado, os resultados confirmaram o comportamento de deformação relatado por Saranath et al. [1]. Portanto, pode-se relacionar os resultados obtidos neste estudo com o que possivelmente aconteceu na microestrutura de cada região da junta devido ao processo de soldagem.

Analisando a figura 5-A, nota-se que a ZAC, representada em roxo, obteve uma deformação de $0,5 \mathrm{~mm} / \mathrm{mm}$, valor bastante considerável em relação ao $\mathrm{MB}$, representado em verde claro, que deformou em média $0,2 \mathrm{~mm} / \mathrm{mm}$.

Esse resultado confirma o que foi reportado por Tolephi et al. [6] e Zhu et al. [7], que devido ao intenso aporte térmico da soldagem aliado ao resfriamento lento resulta no crescimento grosseiro e alongado dos grãos, fato que muito provavelmente promoveu um aumento em ductilidade que gerou o comportamento de deformação observado através da Correlação Digital de Imagens, comparando as regiões MB e ZAC.

A figuras 6-A e 6-B ilustram respectivamente os campos de deformação na direção 2 para os ensaios A2 e B1 nos instantes 580 e 1095s imediatamente antes da ruptura em relação à imagem de referência antes de qualquer deformação.

Notou-se nas figuras 5-A e 6-A, que as regiões do MS apresentaram deformação próximas de zero (cor lilás) nas direções 1 e 2, fato explicado pela presença do reforço dos cordões de solda. Comparando as deformações de MB e MS, observouse que em ambas direções os comportamentos foram relativamente semelhantes, ou seja, o metal de base deformou mais que o metal de solda. 
Assim como observado na figura 5-B, a figura 6-B mostrou que a deformação ao longo do comprimento útil do material não soldado pode ser considerado homogêneo, com aproximadamente $0,193 \mathrm{~mm} / \mathrm{mm}$ de deformação vertical e 0,06 $\mathrm{mm} / \mathrm{mm}$ de deformação horizontal.

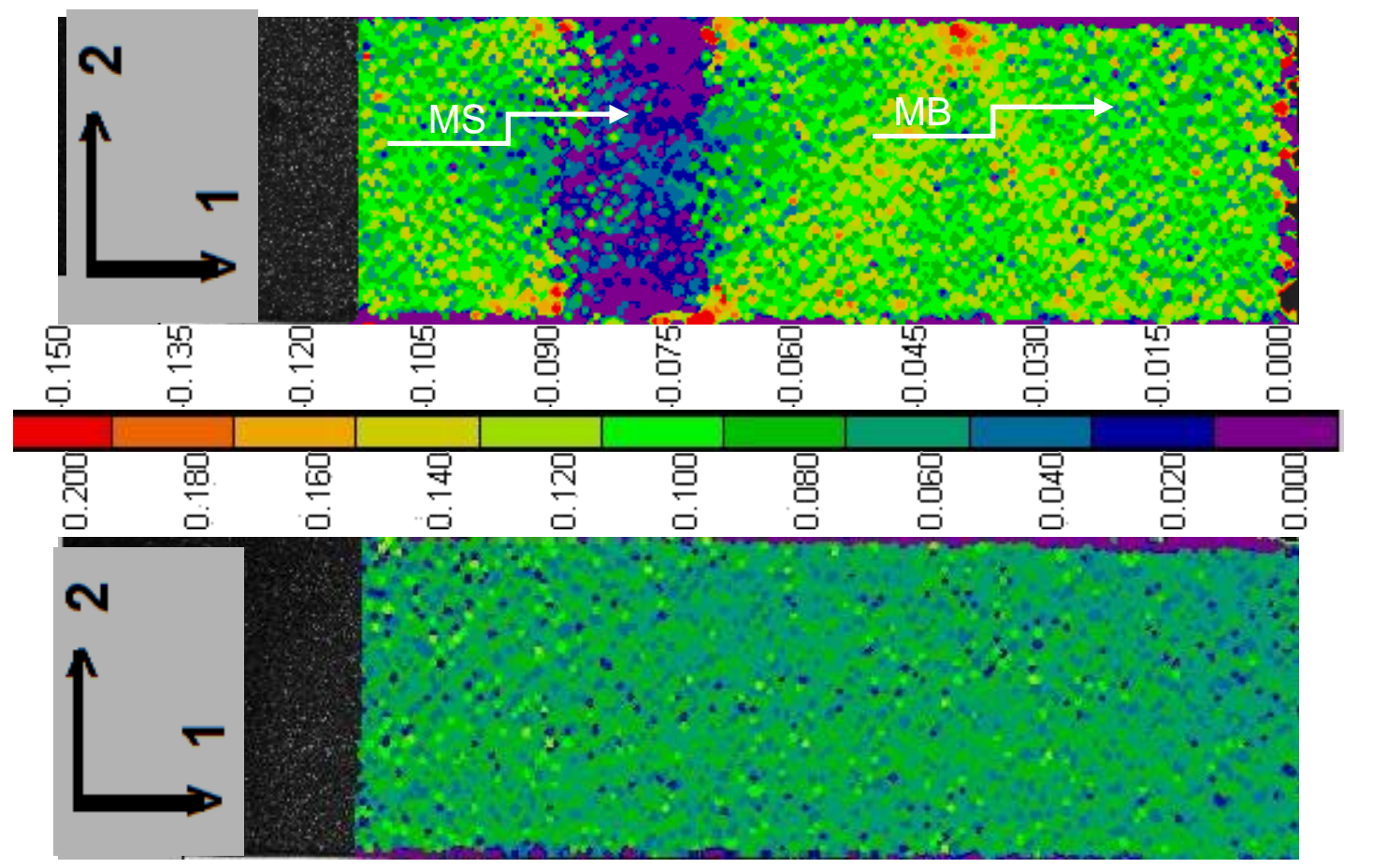

Figura 6- A - Campo de deformação na direção 2, amostra soldada A2 (580s).

Figura 6-B- Campo de deformação na direção 2, amostra não-soldada B1 (1095s).

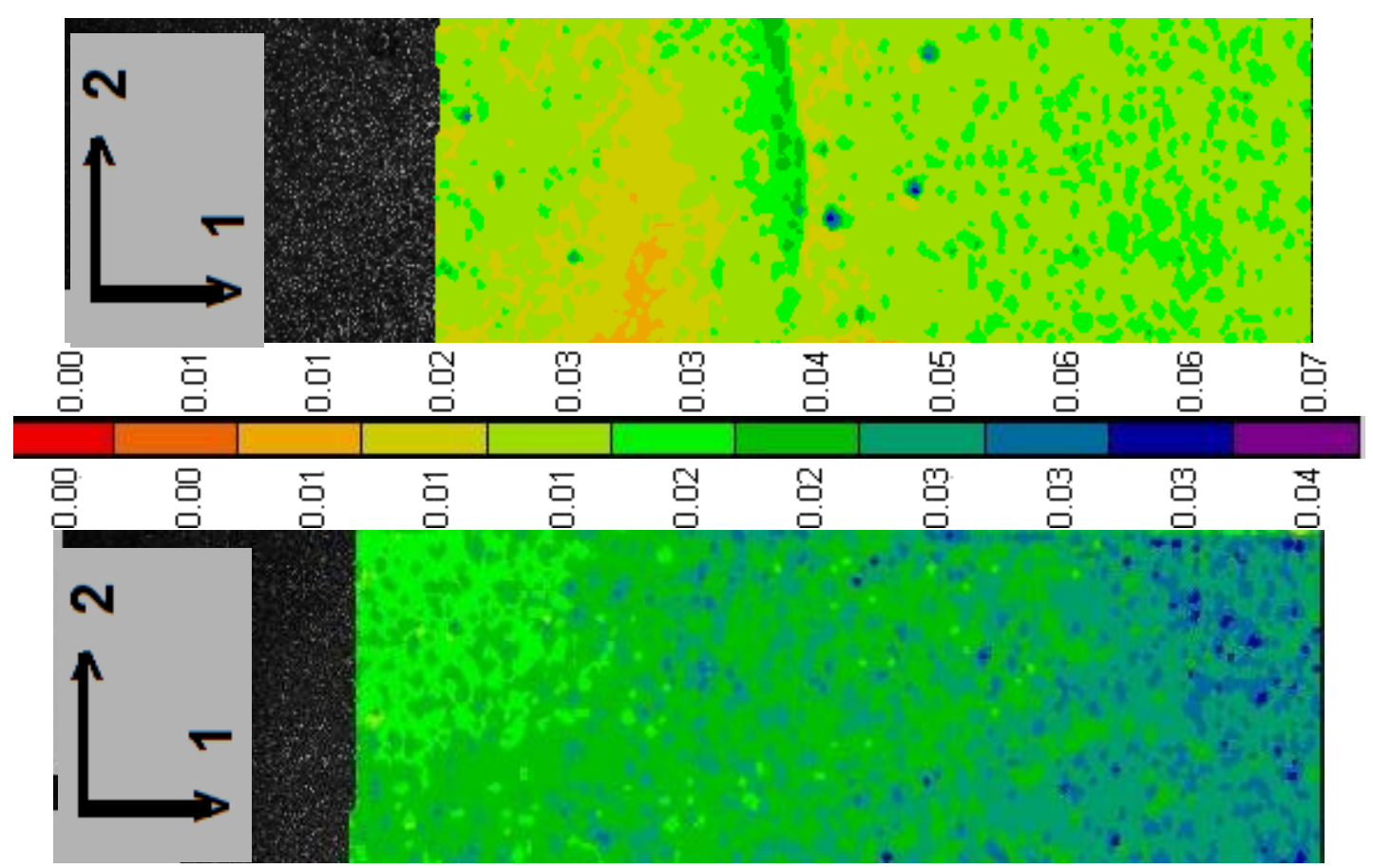

Figura 7-A - Erro amostra soldada A2. Figura 7-B - Amostra não soldada B1. 
A figura 7 mostra a distribuição de erro das correlações. Ambos ensaios obtiveram um erro em torno de 2 a $3 \%$.

\section{CONCLUSÃO}

Através da técnica de Correlação Digital de Imagens foi possível observar o comportamento mecânico de deformação de uma junta soldada de aço inoxidável AISI 304 fazendo a comparação com amostras do mesmo material não-soldado submetidas às mesmas condições de carregamento.

Foi possível identificar o comportamento em cada região da junta, relacionando os subcampos de deformação mostrados com o que diz a literatura sobre as modificações microestruturais decorrentes do aporte térmico da soldagem e sua relação com a alteração do comportamento mecânico de deformação dessas zonas. Adicionalmente, este trabalho confirmou a acuracidade da técnica CDI em identificar campos de deformação, mesmo em um material com intensas modificações microestruturais, que resultou num comportamento de deformação completamente distinto entre as regiões da solda, e ainda comparar com o material com deformação homogênea.

Pode-se concluir que na soldagem do material em estudo a ZAC de grãos grosseiros deformou-se praticamente duas vezes mais que o $\mathrm{MB}$, portanto é a região que possui maior modificação do comportamento mecânico em relação ao material em sua condição original, valendo portanto, um estudo mais aprofundado de seu comportamento mecânico em trabalhos posteriores.

\section{Agradecimentos}

Ao CNPq pelo fomento à pesquisa e ao PET Eng. Mecânica \& Energia e FNDE pelo financiamento das bolsas.

\section{REFERÊNCIAS}

1 SARANATH, K.M.; SHARMA, Abhay; RAMJI, M.. Zone wise local characterization of welds using digital image correlation technique. OPTICS AND LASERS IN ENGINEERING, Hyderabad, India, v. 63, n. 4, p.30-42. 2014.

2 COULES, H. E. et al. High pressure rolling of low carbon steel weld seams: Part 1. SCIENCE AND TECHNOLOGY OF WELDING AND JOINING, Cranfield, p.7683,2013.

3 GALVIS E, A. R.; HORMAZA, W.. Characterization of failure modes for different welding processes of AISI/SAE 304 stainless steels. ENGINEERING FAILURE ANALYSIS, v. 18, n. 7, p.1791-1799,2011.

4 KUMAR, S.; SHAHI, A.S.. Effect of heat input on the microstructure and mechanical properties of gas tungsten arc welded AISI 304 stainless steel joints. MATERIALS \& DESIGN, v. 32, n. 6, p.3617-3623, 2011.

5 CHEN, B.Q., GARBATOV, Y. e GUEDES SOARES, C, "Measurement of weld induced deformations in three-dimensional structures based on photogrammetry technique", Journal of Ship Production and Design, Vol. 27, 2, pp. 51-62. 2011.

6 TOLEPHIH, M. H.; MASHLOOSH, K. M.; WAHEED, Z.. Comparative Study of the Mechanical Properties of (FS) and MIG Welded Joint in (AA7020-T6) Aluminum Alloy. AL-KHWARIZMI ENGINEERING JOURNAL, Baghdad, v. 7, n. 2, p.22-35,2011.

$7 \quad$ ZHU, Z.y. et al. Effect of post weld heat treatment on the microstructure and corrosion behavior of AA2219 aluminum alloy joints welded by variable polarity tungsten inert gas welding. MATERIALS \& DESIGN, v. 65, p.1075-1082, 2015. 
8 CHU, T.C., PETERS, W.H., RANSON, W.F., SUTTON, M.A. Application of digital correlation methods to rigid body mechanics. Proc.1982 FALL MEETING OF SESA, pp. 73-77, 1982.

9 PETERS, W.H., RANSON, W.F. Digital imaging techniques in experimental stress analysis. OPTICAL ENGINEERING 21, 432-437, 1982.

10 SUTTON, M.A., CHENG, M., PETERS, W.H., CHAO, Y.J., MCNEILL, S.R. Application of an optimized digital image correlation method to planar deformation analysis. IMAGE VISION COMPUTING 4 (3), 143-150, 1986.

11 SUTTON, M.A., WOLTERS, W.J., PETERS, W.H., RANSON, W.F., MCNEILL, S.R. Determination of displacements using an improved digital image correlation method. Image Vision Computing 1 (3), 133-139, 1983.

12 BRUCK, H.A., MCNEIL, S.R., SUTTON, M.A., PETERS, W.H. Digital image correlation using Newton-Raphson method of partial differential correction. EXPERIMENTAL MECHANICS 29, 261-267,1989.

$13 \mathrm{HU}$, Hao et al. Image correlation method for full-field deformation measurements during metal sheet welding processes.OPTIK, Shaanxi, p.5193-5198, 2013.

14 HILD F., ROUX S., CORRELIQ4: A software for "finite-element" displacement field measurements by digital image correlation. INTERNAL REPORT n. 269, Cachan, 2008.

15 HILD, F. ROUX, S, Digital Image Correlation: from Displacement Measurement to Identification of Elastic Properties - a Review. STRAIN, 42: 69-80. Cachan, 2006.

16 NAKATA, Toshiya; TANIGAWA, Hiroyasu. Evaluation of local deformation behavior accompanying fatigue damage in $\mathrm{F} 82 \mathrm{H}$ welded joint specimens by using digital image correlation. FUSION ENGINEERING AND DESIGN, Rokkasho, v. 87, n. 6, p.589$593,2012$.

17 Z-Z. Tang, J. Liang, C. Guo, Y.-X. Wang, OPTICAL ENGINEERING. 51, 22012.

18 PENNAFORT, L. C.G. et al. Mechanical characterization of composite PVC/coconut fiber by methods of Acoustic Emission and Digital Image Correlation. SCIENCE AND TECHNOLOGY OF MATERIALS, Fortaleza, v. 80, n. 1, p.1-9, 2014.

19 STAMBORSKÁ, M. et al. Identification of the Stress Fields from the Strain Fields in the Isotropic Materials. PROCEDIA ENGINEERING, v. 48, p.665-672, 2012.

20 PARK, S. et al. Grain Deformation and Strain in Board Level SnAgCu Solder Interconnects Under Deep Thermal Cycling. IEEE TRANSACTIONS ON COMPONENTS AND PACKAGING TECHNOLOGIES, v. 30, n. 1, p.178-185, 2007.

21 STRYCKER, M. et al. Measuring Welding Deformations with the Digital Image Correlation Technique. WELDING JOURNAL,Ghent, v. 90, n. 7, p.107-112, 2011.

22 JIN, T.I.; HA, N.s.; GOO, N.s.. A study of the thermal buckling behavior of a circular aluminum plate using the digital image correlation technique and finite element analysis. THIN-WALLED STRUCTURES, v. 77, p.187-197, 2014.

23 RÊGO, J.P.F.S.; CODES, R.N. Effects of strain rate on thermoplastics materials: an experimental analysis applying digital image correlation. COBEM 2013, Ribeirão Preto SP, nov. 2013.

24 NOGUEIRA DE Codes, R. et al. Spatial and Temporal Characteristics of Propagating Deformation Bands in AA5182 Alloy at Room Temperature. METALLURGICAL AND MATERIALS TRANSACTIONS, Cachan, v. 41, n. 6, p.44-58, jun. 2011 